\title{
A note on degenerate generalized Laguerre polynomials and Lah numbers
}

\author{
Taekyun Kim', Dmitry V. Dolgy²*, Dae San Kim³ ${ }^{3}$ Hye Kyung Kim and Seong Ho Park
}

\section{"Correspondence:}

dvdolgy@gmail.com

${ }^{2}$ Department of Mathematical

Methods in Economy, Far Eastern

Federal University, 690950

Vladivostok, Russia

Full list of author information is

available at the end of the article

\begin{abstract}
The aim of this paper is to introduce the degenerate generalized Laguerre polynomials as the degenerate version of the generalized Laguerre polynomials and to derive some properties related to those polynomials and Lah numbers, including an explicit expression, a Rodrigues type formula, and expressions for the derivatives. The novelty of the present paper is that it is the first paper on degenerate versions of orthogonal polynomials.
\end{abstract}

MSC: 11B83; 42C05; 60E99

Keywords: Degenerate generalized Laguerre polynomials; Lah numbers; Degenerate exponential function

\section{Introduction}

The generalized Laguerre polynomials are classical orthogonal polynomials which are orthogonal with respect to the gamma distribution $e^{-x} x^{\alpha} d x$ on the interval $(0, \infty)$. The generalized Laguerre polynomials are widely used in many problems of quantum mechanics, mathematical physics and engineering. In quantum mechanics, the Schrödinger equation for the hydrogen-like atom is exactly solvable by separation of variables in spherical coordinates. The radial part of the wave function is a generalized Laguerre polynomial [14]. In mathematical physics, vibronic transitions in the Franck-Condon approximation can also be described by using Laguerre polynomials [6]. In engineering, the wave equation is solved for the time domain electric field integral equation for arbitrary shaped conducting structures by expressing the transient behaviors in terms of Laguerre polynomials [4].

The aim of this paper is to introduce the degenerate generalized Laguerre polynomials as the degenerate version of the generalized Laguerre polynomials and to derive some properties related to those polynomials and Lah numbers. In more detail, we obtain an explicit formula and a Rodrigues type formula for the degenerate Laguerre polynomials. We also get explicit expressions for the degenerate generalized Laguerre polynomial for $\alpha=-1$, an identity involving Lah numbers, the falling factorial moment of the degenerate Poisson random variable with parameter $\alpha$, and expressions for the derivatives of the degenerate generalized Laguerre polynomials.

We should mention here that degenerate versions of many special numbers and polynomials have been explored and many interesting results have been obtained in recent years

(c) The Author(s) 2021. This article is licensed under a Creative Commons Attribution 4.0 International License, which permits use, sharing, adaptation, distribution and reproduction in any medium or format, as long as you give appropriate credit to the original author(s) and the source, provide a link to the Creative Commons licence, and indicate if changes were made. The images or other third party material in this article are included in the article's Creative Commons licence, unless indicated otherwise in a credit line to the material. If material is not included in the article's Creative Commons licence and your intended use is not permitted by statutory regulation or exceeds the permitted use, you will need to obtain permission directly from the copyright holder. To view a copy of this licence, visit http://creativecommons.org/licenses/by/4.0/. 
$[8,11,12]$. Furthermore, these have been done not only for special numbers and polynomials but also for transcendental functions like gamma functions [10]. The novelty of the present paper is that this is the first paper which treats degenerate versions of orthogonal polynomials. For the rest of this section, we will recall some necessary facts that will be used throughout this paper.

The Laguerre polynomial $L_{n}(x)$ satisfies the second-order linear differential equation

$$
x y^{\prime \prime}+(1-x) y^{\prime}+n y=0 \quad(\text { see }[16])
$$

while the generalized Laguerre polynomial (or the associated Laguerre polynomial) $L_{n}^{(\alpha)}(x)$ satisfies the second-order linear differential equation

$$
x y^{\prime \prime}+(\alpha+1-x) y^{\prime}+n y=0, \quad(\alpha \in \mathbb{R}) .
$$

The Rodrigues formula of the Laguerre polynomial $L_{n}(x)$ is given by

$$
L_{n}(x)=\frac{e^{x}}{n !} \frac{d^{n}}{d x^{n}}\left(e^{-x} x^{n}\right)=\frac{1}{n !}\left(\frac{d}{d x}-1\right)^{n} x^{n}
$$

while that of the generalized Laguerre polynomial $L_{n}^{(\alpha)}(x)$ is given by

$$
\begin{aligned}
L_{n}^{(\alpha)}(x) & =\frac{1}{n !} x^{-\alpha} e^{x} \frac{d^{n}}{d x^{n}}\left(e^{-x} x^{n+\alpha}\right) \\
& =x^{-\alpha} \frac{1}{n !}\left(\frac{d}{d x}-1\right)^{n} x^{n+\alpha} \quad(\text { see }[2,9,16,17]) .
\end{aligned}
$$

The generating function of generalized Laguerre polynomials is given by

$$
\sum_{n=0}^{\infty} L_{n}^{(\alpha)}(x) t^{n}=\frac{1}{(1-t)^{\alpha+1}} e^{-x} \frac{t}{1-t} \quad(\text { see }[9,16,17])
$$

From (3), we get

$$
L_{n}^{(\alpha)}(x)=\sum_{i=0}^{n}(-1)^{i}\left(\begin{array}{c}
n+\alpha \\
n-i
\end{array}\right) \frac{x^{i}}{i !} \quad(\text { see }[9,17]) .
$$

Note that

$$
\begin{aligned}
& L_{0}^{(\alpha)}(x)=1 \\
& L_{1}^{(\alpha)}(x)=-x+(\alpha+1) \\
& L_{2}^{(\alpha)}(x)=\frac{x^{2}}{2}-(\alpha+2) x+\frac{(\alpha+1)(\alpha+2)}{2}, \\
& L_{3}^{(\alpha)}(x)=-\frac{x^{3}}{6}+\frac{\alpha+3}{2} x^{2}-\frac{(\alpha+2)(\alpha+3)}{2} x+\frac{(\alpha+1)(\alpha+2)(\alpha+3)}{6}, \ldots
\end{aligned}
$$

The rising factorial sequence is defined as

$$
\langle x\rangle_{0}=1, \quad\langle x\rangle_{n}=x(x+1) \cdots(x+n-1), \quad(n \geq 1),
$$


while the falling factorial sequence is defined as

$$
(x)_{0}=1, \quad(x)_{n}=x(x-1) \cdots(x-n+1), \quad(n \geq 1),(\text { see }[1-3,5,7-13,15-18]) .
$$

We note that the Lah numbers are defined by

$$
\langle x\rangle_{n}=\sum_{k=0}^{n} L(n, k)(x)_{k}, \quad(n \geq 0),(\text { see }[3,5,8,13,15]) .
$$

From (5), we can easily derive the following equation:

$$
\frac{1}{k !}\left(\frac{t}{1-t}\right)^{k}=\sum_{n=k}^{\infty} L(n, k) \frac{t^{n}}{n !} \quad(\text { see }[3,5,13,17]) .
$$

For any $\lambda \in \mathbb{R}$, the degenerate exponential function is defined by

$$
e_{\lambda}^{x}(t)=\sum_{n=0}^{\infty}(x)_{n, \lambda} \frac{t^{n}}{n !} \quad(\text { see }[10,11]),
$$

where $(x)_{0, \lambda}=1,(x)_{n, \lambda}=x(x-\lambda) \cdots(x-(n-1) \lambda),(n \geq 1)$. For $x=1$, we use the brief notation $e_{\lambda}(t)=e_{\lambda}^{1}(t)$.

\section{Degenerate generalized Laguerre polynomials}

For any $\alpha \in \mathbb{R}$, we consider the degenerate generalized Laguerre polynomials given by

$$
\frac{1}{(1-t)^{\alpha+1}} e_{\lambda}\left(-x \frac{t}{1-t}\right)=\sum_{n=0}^{\infty} L_{n, \lambda}^{(\alpha)}(x) t^{n}, \quad|t|<1 .
$$

From (7), we note that

$$
\begin{aligned}
\frac{1}{(1-t)^{\alpha+1}} e_{\lambda}\left(-x \frac{t}{1-t}\right) & =\frac{1}{(1-t)^{\alpha+1}} \sum_{m=0}^{\infty}(1)_{m, \lambda}(-1)^{m} x^{m} \frac{1}{m !}\left(\frac{t}{1-t}\right)^{m} \\
& =\sum_{m=0}^{\infty}(1)_{m, \lambda}(-1)^{m} x^{m} \frac{1}{m !} t^{m}\left(\frac{1}{1-t}\right)^{m+\alpha+1} \\
& =\sum_{m=0}^{\infty}(1)_{m, \lambda}(-1)^{m} x^{m} \frac{t^{m}}{m !} \sum_{l=0}^{\infty}\left(\begin{array}{c}
m+\alpha+l \\
l
\end{array}\right) t^{l} \\
& =\sum_{n=0}^{\infty}\left(\sum_{m=0}^{n}(1)_{m, \lambda}(-1)^{m} x^{m} \frac{1}{m !}\left(\begin{array}{c}
m+\alpha+n-m \\
n-m
\end{array}\right)\right) t^{n} \\
& =\sum_{n=0}^{\infty}\left(\sum_{m=0}^{n}(1)_{m, \lambda}(-1)^{m} x^{m} \frac{1}{m !}\left(\begin{array}{c}
n+\alpha \\
n-m
\end{array}\right)\right) t^{n} .
\end{aligned}
$$

Therefore, by (8) and (9), we obtain the following theorem.

Theorem 1 For $n \geq 0$, we have

$$
L_{n, \lambda}^{(\alpha)}(x)=\sum_{m=0}^{n}\left(\begin{array}{c}
n+\alpha \\
n-m
\end{array}\right)(-1)^{m}(1)_{m, \lambda} \frac{1}{m !} x^{m} .
$$


Now, by using Theorem 1, we observe that

$$
\begin{aligned}
& \frac{d^{n}}{d x^{n}}\left[x^{\alpha} e_{\lambda}\left(-\frac{a}{x}\right)\right] \\
& =\frac{d^{n}}{d x^{n}}\left[\sum_{k=0}^{\infty}(1)_{k, \lambda}(-a)^{k} \frac{1}{k !} x^{\alpha-k}\right] \\
& =\sum_{k=0}^{\infty}(1)_{k, \lambda} \frac{(-a)^{k}}{k !} \overbrace{(\alpha-k)(\alpha-k-1) \cdots(\alpha-k-n+1)}^{n \text {-times }} x^{\alpha-k-n} \\
& =(-1)^{n} x^{\alpha-n} \sum_{k=0}^{\infty}(1)_{k, \lambda}(-a)^{k} \frac{1}{k !}(k-\alpha)(k-\alpha+1) \cdots(k-\alpha+n-1) x^{-k} \\
& =(-1)^{n} x^{\alpha-n} n ! \sum_{k=0}^{\infty}(1)_{k, \lambda} \frac{(-1)^{k}}{k !}\left(\frac{a}{x}\right)^{k}\left(\begin{array}{c}
k+n-\alpha-1 \\
n
\end{array}\right) \\
& =(-1)^{n} x^{\alpha-n} n ! \sum_{k=0}^{\infty}(1)_{k, \lambda} \frac{(-1)^{k}}{k !}\left(\frac{a}{x}\right)^{k} \sum_{l=0}^{n}\left(\begin{array}{c}
n-\alpha-1 \\
n-l
\end{array}\right)\left(\begin{array}{l}
k \\
l
\end{array}\right) \\
& =(-1)^{n} x^{\alpha-n} n ! \sum_{l=0}^{n}\left(\begin{array}{c}
n-\alpha-1 \\
n-l
\end{array}\right) \sum_{k=l}^{\infty}(1)_{k, \lambda} \frac{(-1)^{k}}{k !}\left(\frac{a}{x}\right)^{k} \frac{k !}{l !(k-l) !} \\
& =(-1)^{n} x^{\alpha-n} n ! \sum_{l=0}^{n}\left(\begin{array}{c}
n-\alpha-1 \\
n-l
\end{array}\right) \sum_{k=0}^{\infty}(1)_{k+l, \lambda}(-1)^{k+l}\left(\frac{a}{x}\right)^{k+l} \frac{1}{l ! k !} \\
& =(-1)^{n} x^{\alpha-n} n ! \sum_{l=0}^{n}\left(\begin{array}{c}
n-\alpha-1 \\
n-l
\end{array}\right)(-1)^{l}\left(\frac{a}{x}\right)^{l} \frac{1}{l !}(1)_{l, \lambda} \sum_{k=0}^{\infty}(1-l \lambda)_{k, \lambda} \frac{(-1)^{k}}{k !}\left(\frac{a}{x}\right)^{k} \\
& =(-1)^{n} x^{\alpha-n} n ! \sum_{l=0}^{n}\left(\begin{array}{c}
n-\alpha-1 \\
n-l
\end{array}\right)(-1)^{l}(1)_{l, \lambda}\left(\frac{a}{x}\right)^{l} \frac{1}{l !} e_{\lambda}^{1-l \lambda}\left(-\frac{a}{x}\right) \\
& =(-1)^{n} x^{\alpha-n} n ! e_{\lambda}\left(-\frac{a}{x}\right) \sum_{l=0}^{n}\left(\begin{array}{c}
n-\alpha-1 \\
n-l
\end{array}\right)(-1)^{l}(1)_{l, \lambda}\left(\frac{a}{x-a \lambda}\right)^{l} \frac{1}{l !} \\
& =(-1)^{n} x^{\alpha-n} n ! e_{\lambda}\left(-\frac{a}{x}\right) L_{n, \lambda}^{(-\alpha-1)}\left(\frac{a}{x-a \lambda}\right) \text {. }
\end{aligned}
$$

Therefore, by (10), we obtain the following theorem.

Theorem 2 For $n \geq 0$, we have

$$
\frac{d^{n}}{d x^{n}}\left[x^{\alpha} e_{\lambda}\left(-\frac{a}{x}\right)\right]=(-1)^{n} x^{\alpha-n} n ! e_{\lambda}\left(-\frac{a}{x}\right) L_{n, \lambda}^{(-\alpha-1)}\left(\frac{a}{x-a \lambda}\right)
$$

By using Leibniz rule and Theorem 1, we have

$$
\begin{aligned}
& \frac{d^{n}}{d x^{n}}\left[e_{\lambda}(-x) x^{n+\alpha}\right] \\
& =\sum_{m=0}^{n}\left(\begin{array}{l}
n \\
m
\end{array}\right)\left[\frac{d^{m}}{d x^{m}} e_{\lambda}(-x)\right]\left[\frac{d^{n-m}}{d x^{n-m}} x^{n+\alpha}\right]
\end{aligned}
$$




$$
\begin{aligned}
& =\sum_{m=0}^{n}\left(\begin{array}{c}
n \\
m
\end{array}\right)(-1)^{m}(1)_{m, \lambda} e_{\lambda}^{1-m \lambda}(-x) \cdot(n+\alpha)_{n-m} x^{n+\alpha-n+m} \\
& =n ! e_{\lambda}(-x) x^{\alpha} \sum_{m=0}^{n}\left(\begin{array}{c}
n+\alpha \\
n-m
\end{array}\right)(-1)^{m}(1)_{m, \lambda} e_{\lambda}^{-m \lambda}(-x) x^{m} \frac{1}{m !} \\
& =n ! e_{\lambda}(-x) x^{\alpha} \sum_{m=0}^{n}\left(\begin{array}{c}
n+\alpha \\
n-m
\end{array}\right)(-1)^{m}(1)_{m, \lambda}\left(\frac{x}{1-\lambda x}\right)^{m} \frac{1}{m !} \\
& =n ! e_{\lambda}(-x) x^{\alpha} L_{n, \lambda}^{(\alpha)}\left(\frac{x}{1-\lambda x}\right) .
\end{aligned}
$$

Thus, we obtain Rodrigues type formula for the degenerate generalized Laguerre polynomials.

Theorem 3 (Rodrigues type formula) For $n \geq 0$, we have

$$
\frac{x^{-\alpha}}{n ! e_{\lambda}(-x)} \frac{d^{n}}{d x^{n}}\left[e_{\lambda}(-x) x^{n+\alpha}\right]=L_{n, \lambda}^{(\alpha)}\left(\frac{x}{1-\lambda x}\right) .
$$

For $\alpha=-1$, from Theorem 3, we have

$$
\frac{x}{n ! e_{\lambda}(-x)} \frac{d^{n}}{d x^{n}}\left[e_{\lambda}(-x) x^{n-1}\right]=L_{n, \lambda}^{(-1)}\left(\frac{x}{1-x}\right) .
$$

On the other hand, by (8), we get

$$
e_{\lambda}\left(-x \frac{t}{1-t}\right)=\sum_{n=0}^{\infty} L_{n, \lambda}^{(-1)}(x) t^{n}
$$

From (7), we can derive the following equation:

$$
\begin{aligned}
e_{\lambda}\left(-x \frac{t}{1-t}\right) & =\sum_{k=0}^{\infty}(-1)^{k}(1)_{k, \lambda} x^{k} \frac{1}{k !}\left(\frac{t}{1-t}\right)^{k} \\
& =\sum_{k=0}^{\infty}(-1)^{k}(1)_{k, \lambda} x^{k} \sum_{n=k}^{\infty} L(n, k) \frac{t^{n}}{n !} \\
& =\sum_{n=0}^{\infty}\left(\sum_{k=0}^{n}(-1)^{k}(1)_{k, \lambda} x^{k} L(n, k)\right) \frac{t^{n}}{n !} .
\end{aligned}
$$

Thus, by (13) and (14), we get

$$
L_{n, \lambda}^{(-1)}(x)=\frac{1}{n !} \sum_{k=0}^{n}(-x)^{k}(1)_{k, \lambda} L(n, k)
$$

where $L(n, k)=\left(\begin{array}{c}n-1 \\ k-1\end{array}\right) \frac{n !}{k !}$ is the Lah number.

Therefore, we obtain the following theorem. 
Theorem 4 For $n \geq 0$, we have

$$
L_{n, \lambda}^{(-1)}(x)=\frac{1}{n !} \sum_{k=0}^{n}(-x)^{k}(1)_{k, \lambda} L(n, k)=\sum_{k=0}^{n}(1)_{k, \lambda}(-x)^{k} \frac{1}{k !}\left(\begin{array}{l}
n-1 \\
k-1
\end{array}\right) .
$$

From Theorem 1, we note that

$$
\begin{aligned}
L_{n, \lambda}^{(\alpha)}(x) & =\sum_{m=0}^{n}(1)_{m, \lambda} x^{m}(-1)^{m} \frac{1}{m !}\left(\begin{array}{c}
n+\alpha \\
n-m
\end{array}\right) \\
& =\sum_{m=0}^{n}(1)_{m, \lambda}(-x)^{m} \frac{(n+\alpha)(n+\alpha-1) \cdots(m+\alpha+1)}{m !(n-m) !} \\
& =\sum_{m=0}^{n}(1)_{m, \lambda}(-x)^{m} \frac{(n+\alpha)(n+\alpha-1) \cdots(m+\alpha+1)(m+\alpha) \cdots(\alpha+1)}{m !(n-m) !(m+\alpha) \cdots(\alpha+1)} \\
& =\sum_{m=0}^{n}(1)_{m, \lambda}(-x)^{m} \frac{1}{m !(n-m) !} \frac{(n+\alpha)_{n}}{(m+\alpha)_{m}} \\
& =\sum_{m=0}^{n}(1)_{m, \lambda}(-x)^{m} \frac{1}{m !(n-m) !} \frac{(n+\alpha)_{n}}{(m+\alpha)_{m}} \frac{\Gamma(\alpha+1)}{\Gamma(\alpha+1)} \\
& =\Gamma(n+\alpha+1) \sum_{m=0}^{n}(1)_{m, \lambda} \frac{(-x)^{m}}{m !(n-m) !} \frac{1}{\Gamma(m+\alpha+1)} .
\end{aligned}
$$

Thus, by (16), we get

$$
L_{n, \lambda}^{(\alpha)}(x)=\frac{\Gamma(n+\alpha+1)}{\Gamma(n+1)} \sum_{m=0}^{n}(1)_{m, \lambda}\left(\begin{array}{l}
n \\
m
\end{array}\right)(-x)^{m} \frac{1}{\Gamma(m+\alpha+1)} .
$$

In particular, $\alpha=-1$, we have

$$
\begin{aligned}
L_{n, \lambda}^{(-1)}(x) & =\frac{1}{n} \sum_{m=0}^{n}(1)_{m, \lambda}\left(\begin{array}{c}
n \\
m
\end{array}\right)(-x)^{m} \frac{1}{\Gamma(m)} \\
& =\sum_{m=1}^{n}(1)_{m, \lambda}(-x)^{m} \frac{1}{m !}\left(\begin{array}{c}
n-1 \\
m-1
\end{array}\right) .
\end{aligned}
$$

Now, we observe that

$$
\begin{aligned}
\frac{d^{n}}{d x^{n}} e_{\lambda}\left(\frac{1}{x}\right) & =\frac{d^{n}}{d x^{n}} \sum_{k=0}^{\infty}(1)_{k, \lambda} \frac{1}{k !}\left(\frac{1}{x}\right)^{k} \\
& =\sum_{k=0}^{\infty}(1)_{k, \lambda} \frac{1}{k !}(-1)^{n}\langle k\rangle_{n} x^{-n-k} \\
& =\sum_{k=0}^{\infty}(1)_{k, \lambda} \frac{(-1)^{n}}{k !} \sum_{l=0}^{n} L(n, l)(k)_{l} x^{-n-k} \\
& =\sum_{l=0}^{n}(-1)^{n} L(n, l) \sum_{k=0}^{\infty}(1)_{k, \lambda} \frac{(k)_{l}}{k !} x^{-n-k}
\end{aligned}
$$




$$
\begin{aligned}
& =(-1)^{n} x^{-n} \sum_{l=0}^{n} L(n, l) \sum_{k=l}^{\infty} \frac{(1)_{k, \lambda}}{k !}(k)_{l} x^{-k} \\
& =(-1)^{n} x^{-n} \sum_{l=0}^{n} L(n, l) \sum_{k=l}^{\infty} \frac{k !}{k !(k-l) !}(1)_{k, \lambda} x^{-k} \\
& =(-1)^{n} x^{-n} \sum_{l=0}^{n} L(n, l) x^{-l} \sum_{k=0}^{\infty} \frac{(1)_{k+l, \lambda}}{k !} x^{-k} \\
& =(-1)^{n} x^{-n} \sum_{l=0}^{n} L(n, l) x^{-l}(1)_{l, \lambda} \sum_{k=0}^{\infty} \frac{(1-l \lambda)_{k, \lambda}}{k !} x^{-k} \\
& =(-1)^{n} x^{-n} \sum_{l=0}^{n} L(n, l) x^{-l}(1)_{l, \lambda} e_{\lambda}^{1-l \lambda}\left(\frac{1}{x}\right) \\
& =(-1)^{n} x^{-n} e_{\lambda}\left(\frac{1}{x}\right) \sum_{l=0}^{n} L(n, l)(1)_{l, \lambda} x^{-l}\left(1+\frac{\lambda}{x}\right)^{-l} \\
& =(-1)^{n} x^{-n} e_{\lambda}\left(\frac{1}{x}\right) \sum_{l=0}^{n} L(n, l)(1)_{l, \lambda}\left(\frac{1}{x+\lambda}\right)^{l} .
\end{aligned}
$$

Therefore, by (19), we obtain the following theorem.

Theorem 5 For $n \geq 1$, we have

$$
\frac{d^{n}}{d x^{n}} e_{\lambda}\left(\frac{1}{x}\right)=(-1)^{n} x^{-n} e_{\lambda}\left(\frac{1}{x}\right) \sum_{l=0}^{n} L(n, l)(1)_{l, \lambda}\left(\frac{1}{x+\lambda}\right)^{l}
$$

Since

$$
L(n, k)=\left(\begin{array}{l}
n-1 \\
k-1
\end{array}\right) \frac{n !}{k !}=\left(\begin{array}{l}
n-1 \\
k-1
\end{array}\right) \frac{n !}{(n-k) ! k !}(n-k) !=\left(\begin{array}{l}
n-1 \\
k-1
\end{array}\right)\left(\begin{array}{l}
n \\
k
\end{array}\right)(n-k) !
$$

we have the following corollary.

Corollary 6 For $n \geq 1$, we have

$$
\frac{d^{n}}{d x^{n}} e_{\lambda}\left(\frac{1}{x}\right)=x^{-n}(-1)^{n} e_{\lambda}\left(\frac{1}{x}\right) \sum_{l=1}^{n}\left(\begin{array}{c}
n-1 \\
l-1
\end{array}\right)\left(\begin{array}{l}
n \\
l
\end{array}\right)(1)_{l, \lambda}(n-l) !\left(\frac{1}{x+\lambda}\right)^{l}
$$

\section{Degenerate Poisson random variables}

Let $X$ be the Poisson random variable with parameter $\alpha(>0)$. Then the probability mass function of $X$ is given by

$$
p(i)=P\{X=i\}=\frac{\alpha^{i}}{i !} e^{-\alpha} \quad(i=0,1,2, \ldots)
$$

It is easy to show that

$$
E\left[(X)_{n}\right]=\sum_{k=0}^{\infty}(k)_{n} p(k)=e^{-\alpha} \sum_{k=n}^{\infty} \frac{\alpha^{k}}{(k-n) !}=e^{-\alpha} \alpha^{n} \sum_{k=0}^{\infty} \frac{\alpha^{k}}{k !}=\alpha^{n} .
$$


Thus, we note that

$$
E\left[\left(\begin{array}{l}
X \\
n
\end{array}\right)\right]=\frac{\alpha^{n}}{n !} \quad(n=0,1,2, \ldots) .
$$

Let $X_{\lambda}$ be the degenerate Poisson random variable with parameter $\alpha(>0)$. Then the probability mass function of $X_{\lambda}$ is given by

$$
p(i)=P\left\{X_{\lambda}=i\right\}=e_{\lambda}^{-1}(\alpha) \frac{\alpha^{i}}{i !}(1)_{i, \lambda} \quad(i=0,1,2, \ldots),(\text { see }[12]) .
$$

Then the following falling factorial moment is given by

$$
\begin{aligned}
E\left[\left(X_{\lambda}\right)_{n}\right] & =\sum_{k=0}^{\infty}(k)_{n} p(k)=\sum_{k=0}^{\infty}(k)_{n} \frac{e_{\lambda}^{-1}(\alpha)}{k !} \alpha^{k}(1)_{k, \lambda} \\
& =e_{\lambda}^{-1}(\alpha) \sum_{k=n}^{\infty} \frac{k(k-1) \cdots(k-n+1)(k-n) !}{k !(k-n) !} \alpha^{k}(1)_{k, \lambda} \\
& =e_{\lambda}^{-1}(\alpha) \sum_{k=0}^{\infty} \alpha^{k+n} \frac{1}{k !}(1)_{k+n, \lambda} \\
& =\alpha^{n} e_{\lambda}^{-1}(\alpha) \sum_{k=0}^{\infty}(1)_{n, \lambda}(1-n \lambda)_{k, \lambda} \frac{\alpha^{k}}{k !} \\
& =\alpha^{n} e_{\lambda}^{-1}(\alpha)(1)_{n, \lambda} e_{\lambda}^{1-n \lambda}(\alpha)=\alpha^{n}(1)_{n, \lambda}\left(\frac{1}{1+\alpha \lambda}\right)^{n} .
\end{aligned}
$$

Assume that $X_{\lambda}$ is the Poisson random variable with parameter $\frac{1}{\alpha}(>0)$. Then, by using (20), we obtain

$$
\begin{aligned}
\frac{d^{n}}{d \alpha^{n}}\left(e_{\lambda}\left(\frac{1}{\alpha}\right)\right) & =(-1)^{n} \alpha^{-n} \sum_{l=0}^{n} L(n, l) \sum_{k=0}^{\infty} \frac{(k)_{l}}{k !}(1)_{k, \lambda} \alpha^{-k} \\
& =(-1)^{n} \alpha^{-n} \sum_{l=0}^{n} L(n, l) e_{\lambda}\left(\frac{1}{\alpha}\right) e_{\lambda}^{-1}\left(\frac{1}{\alpha}\right) \sum_{k=0}^{\infty} \frac{(k)_{l}}{k !}(1)_{k, \lambda} \alpha^{-k} \\
& =(-1)^{n} \alpha^{-n} \sum_{l=0}^{n} L(n, l) e_{\lambda}\left(\frac{1}{\alpha}\right) E\left[\left(X_{\lambda}\right)_{l}\right] \\
& =(-1)^{n} \alpha^{-n} e_{\lambda}\left(\frac{1}{\alpha}\right) \sum_{l=0}^{n} L(n, l)(1)_{l, \lambda}\left(\frac{1}{\alpha+\lambda}\right)^{l} .
\end{aligned}
$$

\section{Derivatives of degenerate Laguerre polynomials}

Let us consider the sequence $y_{n, \lambda}(x)$ which is given by

$$
A(t) e_{\lambda}\left(-x \frac{t}{1-t}\right)=\sum_{n=0}^{\infty} y_{n, \lambda}(x) t^{n}
$$

where $A(t)$ is an invertible series. 
Note that $y_{0, \lambda}(x)=A(0)$ is a constant. We now set $F_{\lambda}=F_{\lambda}(x, t)=A(t) e_{\lambda}\left(-\frac{x}{1-t} t\right)$.

From (21), we note that

$$
\begin{aligned}
\frac{\partial}{\partial x} F_{\lambda} & =A(t)\left(-\frac{t}{1-t}\right) e_{\lambda}^{1-\lambda}\left(-\frac{x t}{1-t}\right) \\
& =-\left(\frac{t}{(1-t)-x \lambda t}\right) A(t) e_{\lambda}\left(-\frac{x t}{1-t}\right) .
\end{aligned}
$$

By (22), we get

$$
\frac{\partial}{\partial x} F_{\lambda}-(1+x \lambda) t \frac{\partial}{\partial x} F_{\lambda}=-t F_{\lambda}
$$

From (21) and (23), we can derive the following equation:

$$
\sum_{n=1}^{\infty} y_{n, \lambda}^{\prime}(x) t^{n}-(1+x \lambda) \sum_{n=1}^{\infty} y_{n-1, \lambda}^{\prime}(x) t^{n}=-\sum_{n=1}^{\infty} y_{n-1, \lambda}(x) t^{n} .
$$

By comparing the coefficients on both sides of (24), we get

$$
y_{n, \lambda}^{\prime}(x)-(1+x \lambda) y_{n-1, \lambda}^{\prime}(x)=-y_{n-1, \lambda}(x), \quad(n \geq 1),
$$

where $y_{n, \lambda}^{\prime}(x)=\frac{d}{d x} y_{n, \lambda}(x)$.

Now, we observe that

$$
\begin{aligned}
\frac{-t}{(1-t)-x \lambda t} & =-\frac{t}{1-t}\left(\frac{1}{1-\frac{x \lambda}{1-t} t}\right)=-\sum_{l=0}^{\infty} x^{l} \lambda^{l}\left(\frac{t}{1-t}\right)^{l+1} \\
& =-\sum_{l=1}^{\infty} x^{l-1} \lambda^{l-1}\left(\frac{t}{1-t}\right)^{l} \\
& =-\sum_{l=1}^{\infty} x^{l-1} \lambda^{l-1} t^{l} \sum_{m=0}^{\infty}\left(\begin{array}{c}
m+l-1 \\
m
\end{array}\right) t^{m} \\
& =-\sum_{k=1}^{\infty}\left(\sum_{l=1}^{k} x^{l-1} \lambda^{l-1}\left(\begin{array}{c}
k-1 \\
k-l
\end{array}\right)\right) t^{k} .
\end{aligned}
$$

From (22) and (26), we can derive the following equation:

$$
\begin{aligned}
\sum_{n=1}^{\infty} y_{n, \lambda}^{\prime}(x) t^{n} & =\frac{\partial}{\partial x} F_{\lambda}=-\left(\frac{t}{1-t-x \lambda t}\right) A(t) e_{\lambda}\left(-\frac{x t}{1-t}\right) \\
& =\sum_{k=1}^{\infty}\left(-\sum_{l=1}^{k} x^{l-1} \lambda^{l-1}\left(\begin{array}{c}
k-1 \\
k-l
\end{array}\right)\right) t^{k} \sum_{m=0}^{\infty} y_{m, \lambda}(x) t^{m} \\
& =\sum_{n=1}^{\infty}\left(-\sum_{k=1}^{n} \sum_{l=1}^{k} x^{l-1} \lambda^{l-1}\left(\begin{array}{c}
k-1 \\
k-l
\end{array}\right) y_{n-k, \lambda}(x)\right) t^{n}
\end{aligned}
$$


Thus, by comparing the coefficients on both sides of (27), we get

$$
y_{n, \lambda}^{\prime}(x)=-\sum_{k=1}^{n} \sum_{l=1}^{k} x^{l-1} \lambda^{l-1}\left(\begin{array}{c}
k-1 \\
k-l
\end{array}\right) y_{n-k, \lambda}(x) .
$$

Therefore, we obtain the following theorem.

\section{Theorem 7 Let}

$$
A(t) e_{\lambda}\left(-\frac{x t}{1-t}\right)=\sum_{n=0}^{\infty} y_{n, \lambda}(x) t^{n},
$$

where $A(t)$ is an invertible series.

Then, for $n \geq 1$, we have

$$
y_{n, \lambda}^{\prime}(x)=(1+x \lambda) y_{n-1, \lambda}^{\prime}(x)-y_{n-1, \lambda}(x)
$$

and

$$
y_{n, \lambda}^{\prime}(x)=-\sum_{k=1}^{n} \sum_{l=1}^{k} x^{l-1} \lambda^{l-1}\left(\begin{array}{c}
k-1 \\
k-l
\end{array}\right) y_{n-k, \lambda}(x),
$$

where $y_{n, \lambda}^{\prime}(x)=\frac{d}{d x} y_{n, \lambda}(x)$.

From the definition of the degenerate generalized Laguerre polynomials in (8), we observe that

$$
\begin{aligned}
\sum_{n=0}^{\infty} \frac{d}{d x} L_{n, \lambda}^{(\alpha)}(x) t^{n} & =\frac{1}{(1-t)^{\alpha+1}} \frac{d}{d x} e_{\lambda}\left(-x \frac{t}{1-t}\right) \\
& =-t \frac{1}{(1-t)^{\alpha+2}}\left(1-\frac{\lambda}{1-\lambda}(1-\lambda) x \frac{t}{1-t}\right)^{\frac{1-\lambda}{\lambda}} \\
& =-t \sum_{n=0}^{\infty} L_{n, \frac{\lambda}{1-\lambda}}^{(\alpha+1)}((1-\lambda) x) t^{n} \\
& =-\sum_{n=1}^{\infty} L_{n-1, \frac{\lambda}{1-\lambda}}^{(\alpha+1)}((1-\lambda) x) t^{n} .
\end{aligned}
$$

In Theorem 7, let us take $A(t)=(1-t)^{-\alpha-1}$. Then we have

$$
\sum_{n=0}^{\infty} y_{n, \lambda}(x) t^{n}=(1-t)^{-\alpha-1} e_{\lambda}\left(-\frac{x}{1-x} t\right)=\sum_{n=0}^{\infty} L_{n, \lambda}^{(\alpha)}(x) t^{n} .
$$

Thus, we note that $y_{n, \lambda}(x)=L_{n, \lambda}^{(\alpha)}(x),(n \geq 0)$.

Therefore, by Theorem 7, (29), and (30), we obtain the following corollary.

Corollary 8 For $n \geq 1$, we have the following derivative formulas:

$$
\frac{d}{d x} L_{n, \lambda}^{(\alpha)}(x)=(1+x \lambda) \frac{d}{d x} L_{n-1, \lambda}^{(\alpha)}(x)-L_{n-1, \lambda}^{(\alpha)}(x),
$$




$$
\begin{aligned}
& \frac{d}{d x} L_{n, \lambda}^{(\alpha)}(x)=-\sum_{k=1}^{n} \sum_{l=1}^{k} x^{l-1} \lambda^{l-1}\left(\begin{array}{c}
k-1 \\
k-l
\end{array}\right) L_{n-k, \lambda}^{(\alpha)}(x), \\
& \frac{d}{d x} L_{n, \lambda}^{(\alpha)}(x)=-L_{n-1, \frac{\lambda}{1-\lambda}}^{(\alpha+1)}((1-\lambda) x) .
\end{aligned}
$$

Remark 9 The last derivative formula in Corollary 8 was drawn attention to by one of the referees to whom we thank.

\section{Conclusion}

In this paper, we introduced the degenerate generalized Laguerre polynomials, which are the first degenerate versions of the orthogonal polynomials, and derived some results related to those polynomials and Lah numbers. Some of the results are an explicit expression, Rodrigues type formula, and some expressions for the derivatives of the degenerate generalized Laguerre polynomials.

Acknowledgements

We would like to thank the referees who helped improve the original manuscript in its present form.

Funding

This work was supported by the Basic Science Research Program, the National Research Foundation of Korea (NRF-2021R1F1A1050151).

Availability of data and materials

No data were used to support this study.

Competing interests

The authors declare that they have no competing interests.

Authors' contributions

The authors contributed equally to the work. All authors read and approved the final manuscript.

\section{Author details}

${ }^{1}$ Department of Mathematics, Kwangwoon University, Seoul 139-701, Republic of Korea. ${ }^{2}$ Department of Mathematical Methods in Economy, Far Eastern Federal University, 690950 Vladivostok, Russia. ${ }^{3}$ Department of Mathematics, Sogang University, Seoul 121-742, Republic of Korea. ${ }^{4}$ Department of Mathematics Education, Daegu Catholic University, Gyeongsan 38430, Republic of Korea.

\section{Publisher's Note}

Springer Nature remains neutral with regard to jurisdictional claims in published maps and institutional affiliations.

Received: 6 July 2021 Accepted: 2 September 2021 Published online: 17 September 2021

\section{References}

1. Bayad, A., Chikhi, J.: Apostol-Euler polynomials and asymptotics for negative binomial reciprocals. Adv. Stud. Contemp. Math. (Kyungshang) 24(1), 33-37 (2014)

2. Boyadzhiev, K.N.: Lah numbers, Laguerre polynomials of order negative one, and the $n$th derivative of $\exp (1 / x)$. Acta Univ. Sapientiae Math. 8(1), 22-31 (2016)

3. Carlitz, L. Some generating functions for Laguerre polynomials. Duke Math. J. 35, 825-827 (1968)

4. Chung, Y.-S., Sarkar, T.K., Jung, B.H., Salazar-Palma, M., Ji, Z., Jang, S., Kim, K.: Solution of time domain electric field integral equation using the L polynomials. IEEE Trans. Antennas Propag. 52(9), 2319-2328 (2004)

5. Comtet, L.: Advanced Combinatorics. Reidel, Dordrecht (1974)

6. de Jong, M., Seijo, L., Meijerink, A., Rabouw, F.T.: Resolving the ambiguity in the relation between Stokes shift and Huang-Rhys parameter. Phys. Chem. Chem. Phys. 17(26), 16959-16969 (2015)

7. Khan, W.A., Araci, S., Acikgoz, M., Esi, A.: Laguerre-based Hermite-Bernoulli polynomials associated with bilateral series. Tbil. Math. J. 11(2), 111-121 (2018)

8. Kim, H.K., Lee, D.S.: Some identities of degenerate r-extended Lah-Bell polynomials. Proc. Jangjeon Math. Soc. 24(1), 47-61 (2021)

9. Kim, T.: Identities involving Laguerre polynomials derived from umbral calculus. Russ. J. Math. Phys. 21(1), 36-45 (2014)

10. Kim, T., Kim, D.S.: Note on the degenerate gamma function. Russ. J. Math. Phys. 27(3), 352-358 (2020)

11. Kim, T., Kim, D.S.: Degenerate zero-truncated Poisson random variables. Russ. J. Math. Phys. 28(1), 66-72 (2021)

12. Kim, T., Kim, D.S., Jang, L.-C., Kim, H.Y.: A note on discrete degenerate random variables. Proc. Jangjeon Math. Soc. 23(1), 125-135 (2020) 
13. Kim, T., Kim, D.S., Jang, L.-C., Lee, H., Kim, H.-Y.: Complete and incomplete Bell polynomials associated with Lah-Bell numbers and polynomials. Adv. Differ. Equ. 2021, Paper No. 101, 12 pp. (2021)

14. Mawhin, J., Ronveaux, A.: Schrödinger and Dirac equations for the hydrogen atom, and Laguerre polynomials. Arch. Hist. Exact Sci. 64, 429-460 (2010)

15. Quaintance, J., Gould, H.W.: Combinatorial Identities for Stirling Numbers. World Scientific, Singapore (2016) The unpublished notes of H.W. Gould. With a foreword by George E. Andrews

16. Rainville, E.D.: Special Functions. Chelsea, Bronx (1971) Reprint of 1960 first edition

17. Roman, S.: The Umbral Calculus. Pure and Applied Mathematics, vol. 111. Academic Press, New York (1984)

18. Simsek, Y.: Identities and relations related to combinatorial numbers and polynomials. Proc. Jangjeon Math. Soc. 20(1), 127-135 (2017)

Submit your manuscript to a SpringerOpen ${ }^{\circ}$ journal and benefit from:

- Convenient online submission

- Rigorous peer review

- Open access: articles freely available online

- High visibility within the field

- Retaining the copyright to your article

Submit your next manuscript at $\gg$ springeropen.com 